\title{
Wettability modification of porous PET by atmospheric femtosecond PLD
}

Youssef Assaf ${ }^{\mathrm{a}}$, Guillaume Forstmann ${ }^{\mathrm{a}}$ and Anne-Marie Kietzig ${ }^{\mathrm{a} *}$

${ }^{a}$ Department of Chemical Engineering, McGill University, 3610 University Street, Montreal, Canada, H3A OC5

Youssef Assaf: +1(514) 946-8893; youssef.assaf@mail.mcgill.ca

Guillaume Forstmann: +1(514) 265-8308; guillaume.forstmann@mail.mcgill.ca

*Anne Kietzig (corresponding author): +1(514) 398-3302; anne.kietzig@ mcgill.ca 


\section{Wettability modification of porous PET by atmospheric femtosecond PLD}

In this study, porous structures were created on poly(ethylene terephthalate) (PET) by femtosecond (fs) laser micromachining. While such structures offer a texture that is desirable for several applications, their wettability does not always match the application in question. The aim of this investigation is to tune the wettability of such surfaces by incorporating a controlled amount of nanoparticles into the structure. The machined PET samples were thus used as substrates for fs pulsed laser deposition (PLD) of titanium under ambient conditions. The nanoparticles were deposited as nanochain clusters due to the formation of an oxide layer between individual nanoparticles. The stability of nanoparticle incorporation was tested by placing the samples in an ultrasonic ethanol bath. Results indicated that nanoparticles were still successfully incorporated into the microstructure after sonication. Nanoparticle surface coverage was observed to be controllable through the operating fluence. The dynamic contact angles of the resulting composite surface were observed to decrease with increasing titanium incorporation. Therefore, this work highlights atmospheric fs PLD as a method for wettability modification of high surface area microstructures without undermining their topology. In addition, this technique uses almost the same equipment as the machining process by which the microstructures are initially created, further highlighting its practicality.

Keywords: femtosecond; micromachining; nanoparticle; wettability; PLD

\section{Introduction}

Polymeric materials with high surface area have recently become highly sought after materials due to their wide range of application possibilities including catalysis [1] and biomedical implant materials [2]. They are typically produced by surface modification techniques that induce porosity or a dual-scale texture [3]. Such production methods usually alter the physical and chemical properties of the surface. The resulting material will therefore exhibit specific surface properties that are not easily altered 
without compromising the newly created surface texture, thus limiting its versatility with regards to the application in question. Recently, we have reported on processing polymer surfaces by femtosecond (fs) laser micromachining, which results in porous surface structures that further exhibit nanoscale roughness [4]. Even if the mechanism behind the formation of these surface structures is not yet fully established, they have been observed to appear on a wide variety of polymers upon laser machining [5]. Their novel surface topography makes them prime candidates for various high surface area applications. In this study, we explore the possibility of varying one of the surface properties, namely surface wettability, on a continuous scale with minimal modification to the porous surface topography.

The wettability of a material is dictated by its surface chemistry and roughness. Therefore, wettability modification of a given surface can be achieved by tuning one or both of these properties. In the case of polymers, surface chemistry is typically altered by plasma treatment or UV irradiation by the addition of new functional groups [6]. While such techniques have been reported to successfully alter wettability, they require parameter modification on a case-by-case basis as the outcome heavily depends on the nature of the polymer in question. On the other hand, increasing surface roughness enhances the intrinsic wettability of a homogenous surface as described by the Wenzel wettability model [7].

$$
\cos \theta^{*}=r \cos \theta
$$

Where $\theta$ is the Young's contact angle, $\theta^{*}$ is the apparent contact angle, and $r$ is the roughness factor. One approach for polymer roughness enhancement is to directly impart a microscale pattern onto the surface. Over the years, several techniques have been developed to that effect such as photolithogrpahy [8-10], direct laser texturing [11-13], 
or plasma processing [14-16]. But since fs laser machined polymers are already microtextured, any additional texturing will cause significant damage to the high surface area topology. Therefore, the only way to enhance surface roughness without compromising the underlying microstructure is to modify it on the nanoscale through the incorporation of nanoparticles. This will in fact serve a dual purpose as not only will the added nanoparticles increase the value of the roughness factor $r$ (Equation 1), but they will also induce chemical heterogeneity on the surface as their composition is different than that of the substrate. For such chemically heterogeneous surfaces, the overall wettability will also heavily depend on the relative amount of each component as described by the Cassie-Baxter wettability model for a multi-component system [17].

$$
\cos \theta^{*}=f_{1} \cos \theta_{1}+f_{2} \cos \theta_{2}+\ldots+f_{i} \cos \theta_{i}
$$

Where $\theta_{i}$ is the Young's contact angle of component $i$ and $f_{i}$ is its area fraction. This implies that, if the nanoparticles offer a surface chemistry that is sufficiently distinct from the substrate material, tuneable wettability can be achieved by controlling the density of the deposited nanoparticle coverage [18]. Over the years, many techniques for nanoparticle deposition such as chemical vapour deposition and layer by layer deposition have been developed. However, they typically have complex requirements such as a highly controlled environment and separate nanoparticle generation and deposition steps [19]. Pulsed laser deposition (PLD) is a single step alternative for the simultaneous generation and deposition of nanoparticles by laser ablation of a target material which, in addition, can be performed by using the same equipment as laser micromachining.

In PLD, nanoparticles are generated by focusing an incoming beam onto a target placed in a vacuum chamber causing material ejection through ablation and plasma formation. The expanding nanoparticle plume then reaches the substrate to be coated 
where it condenses and deposits as a uniform thin film. Typically, operating at increased pulse numbers and high fluence increases the impinging nanoparticle flux on the substrate and promotes film formation [20]. The main drawback being that a high flux can increase sputtering damage and promote the inclusion of unwanted microscopic particulates due to melting and exfoliation of the target [21]. With the development of fs laser technology, this effect has been mitigated as the very small timescale of ultrashort pulses minimises thermal effects [22-24]. It is also critical that the nanoparticle plume reaches the substrate in order for deposition to occur. This is why PLD is typically carried out in a vacuum chamber as this minimises the resistance to plasma plume expansion and ensures a working distance in the centimetre range. The need for vacuum is considered one of the main limitations of this technique since it limits cost-effectiveness and sustainability. Yet, a surprisingly low number of studies have attempted to overcome that. Out of the few reports studying atmospheric pressure PLD, the studies by Kononenko et al. [25] and Nedyalkov et al. [26] have successfully demonstrated film formation by working at very high pulse numbers and target-substrate distances in the millimetre range. Similarly, McCann et al. operated at ambient conditions by focusing a laser source through a transparent substrate which was micrometres away from the target [27]. Finally, film formation on the substrate is dominated by the nucleation rate of adsorbed nanoparticle clusters and their subsequent surface diffusion and coalescence into a uniform film [28]. In order to promote surface diffusion, PLD is usually performed on heated substrates with very smooth surfaces [29].

In this study, a porous surface structure was created on poly(ethylene terephthalate) by fs laser micromachining to later serve as a substrate for atmospheric PLD of titanium nanoparticles. While PLD has been used in the past for the deposition of films that exhibit a nanoscale structure [30], it has almost exclusively been used as a coating technique to 
completely cover a smooth, low surface roughness substrate with a uniform film. In our study, we aim at exploiting the high surface roughness of our substrate to actually inhibit surface diffusion and therefore promote the deposition of separate nanoparticle clusters instead of uniform film formation. This is desirable in our case since it will mitigate the possibility of completely covering the underlying porous structure. In addition, the whole process is performed under ambient pressure, thus circumventing the traditional requirement for low pressure conditions. Applying atmospheric PLD for nanoparticle deposition into high-surface area polymer surfaces is therefore highly unconventional and, to the authors' knowledge, unreported yet. One of the main advantages of this technique is that it requires the same equipment as for the machining process by which the polymer surfaces are initially created. The surface coverage density of nanoparticles was manipulated by optimising operating parameters. Finally, the resulting change in wettability caused by the changes in surface chemistry and roughness was measured by X-ray photoelectron spectroscopy (XPS) and dynamic contact angle measurements.

\section{Material and methods}

\subsection{Materials}

For PET, 4 by $4 \mathrm{~mm}$ square samples were cut from a $1.5 \mathrm{~mm}$ thick commercially available sheet (McMaster-Carr, USA). The sides were polished with 1200 grit silicon carbide paper in a grinder/polisher (Leco SS-200, USA) in order to improve baseline accuracy during advancing and receding contact angle measurements. Similarly, 1 by 1 $\mathrm{cm}$ square titanium samples were cut from a sheet with a thickness of $0.5 \mathrm{~mm}$ (McMasterCarr, USA).

\subsection{Laser system}

Micromachining and PLD were performed using an amplified Ti:sapphire laser (Coherent Libra, USA) which emits a horizontally polarised Gaussian beam of $800 \mathrm{~nm}$ 
wavelength, $4 \mathrm{~W}$ output power, in pulses of $150 \mathrm{fs}$ pulse duration, and at a repetition rate of $1 \mathrm{kHz}$. The beam power is controlled through a variable attenuator which consists of a half-wave plate and a polarising beam splitter. The beam is then focused using a $100 \mathrm{~mm}$ plano-convex lens down to a theoretical $1 / e^{2}$ spot size of $44 \mu \mathrm{m}$ onto a motorised 3D translational stage (Newport Corporation, USA) which is subjected to the stationary laser beam. The stage's movement and velocity were digitally controlled via the GOL3D software (GBC\&S, France) along with a mechanical shutter (Uniblitz, USA).

\subsection{Porous PET surface preparation}

The porous, high surface area microstructures that served as substrates for PLD were created by femtosecond laser micromachining of the PET samples at focus. As outlined in our previous work [5], the machining procedure involves simple raster scanning of the laser beam on the sample surface at a single pulse peak fluence $F_{0}=3.9$ $\mathrm{J} / \mathrm{cm}^{2}$ with 55 pulses-per-spot $(P P S)$. Figure 1 illustrates the resulting increase in surface roughness and porosity. Immediately after machining, all samples were placed in a sonication ethanol bath for five minutes to remove any loose machining debris.

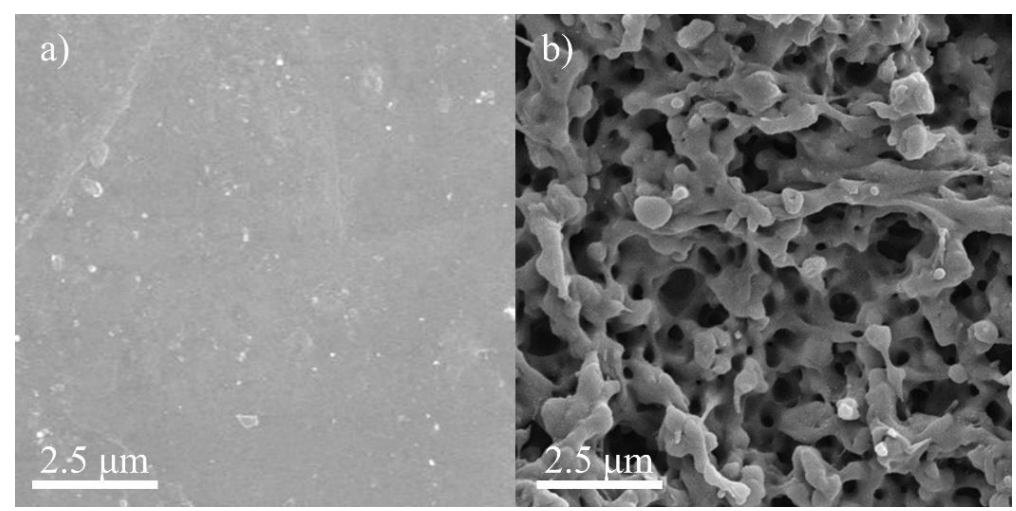

Figure 1: Comparison between a) pristine and b) micromachined PET.

\subsection{Atmospheric PLD setup}

The pulsed laser deposition setup is illustrated in Figure 2. The titanium target and surface modified PET substrate were placed in parallel at an adjustable distance $d$ from each other and at a $45^{\circ}$ angle with respect to the incoming beam. Titanium ablation was 
performed by linearly translating the sample holder with respect to the stationary beam over a distance of $5 \mathrm{~mm}$ in order to cover all of the substrate. The ablated line was overscanned several times with the overscan number $n$ and scanning velocity $v$ used as adjustable operating parameters. Finally, for each sample, the whole process was repeated 4 times while rotating the substrate by $90^{\circ}$ in between each run in order to ensure homogeneous nanoparticle deposition.

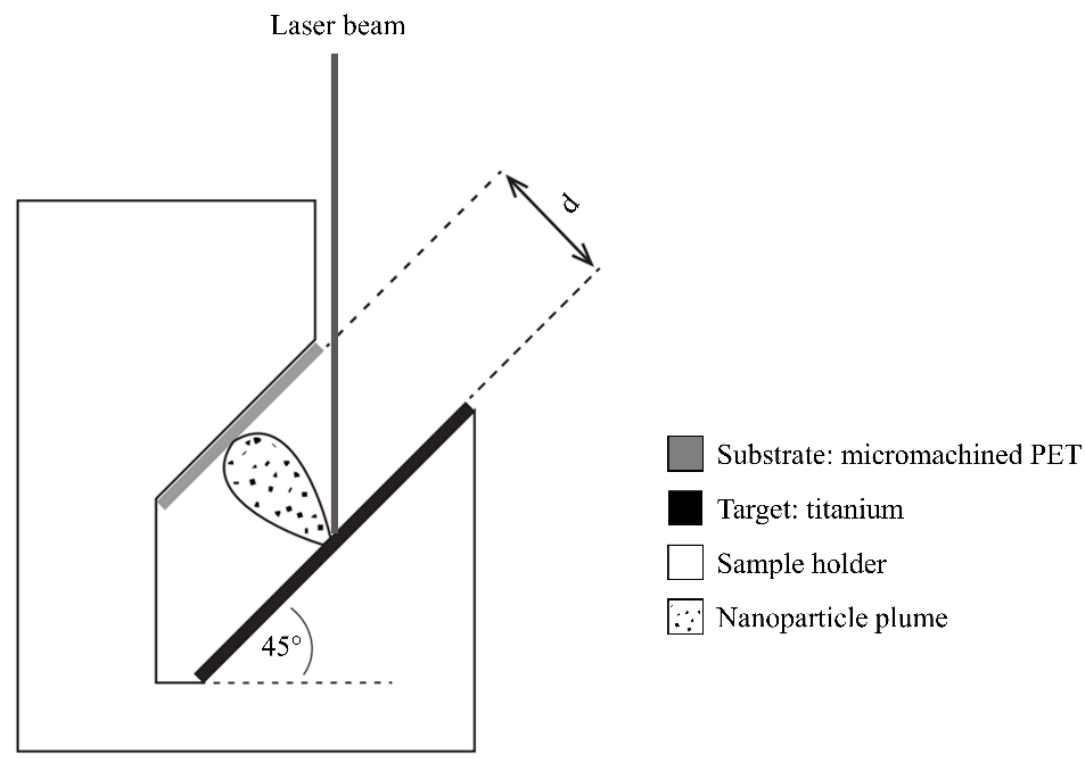

Figure 2: Schematic of PLD setup.

\subsection{Surface analysis}

Advancing $\left(\theta_{A}\right)$ and receding $\left(\theta_{R}\right)$ contact angles were measured at room temperature using a goniometer (DataPhysics OCA 15EC, USA). An initial droplet size of $2 \mu \mathrm{L}$ of filtered reverse osmosis water $(<10 \mathrm{ppb}$ dissolved solids, $1-2 \mu \mathrm{S} / \mathrm{cm}$ conductivity) was used in the measurements. The droplet size was increased to $4 \mu \mathrm{L}$ with a dispense rate of $0.1 \mu \mathrm{L} / \mathrm{s}$ and then reduced back to the original volume at the same rate. Three separate measurements were performed for each sample.

Chemical characterisation of the various films was performed via X-ray photoelectron spectroscopy (XPS) (ThermoFisher scientific K-alpha, USA). Measurements were taken using a $400 \mu \mathrm{m}$ spot size with an Argon ion flood gun turned 
on in order to compensate for surface charging. Two types of scans were performed for each sample. A survey scan (200 eV pass energy, 3 scans, $10 \mathrm{~ms}$ dwell time) in order to determine composition and high resolution scans $(50 \mathrm{eV}$ pass energy, $10 \mathrm{scans}, 50 \mathrm{~ms}$ dwell time) for the 1s sublevel of Carbon and Oxygen and the $2 p$ sublevel of titanium in order to determine bonding structure.

The surface topography was imaged via scanning electron microscopy (SEM) (FEI Inspect F50, USA). This required coating the samples with a very thin (1-2 nm) layer of gold by low-vacuum sputtering in order to avoid surface charging.

\section{Results and discussion}

\subsection{Effect of target-substrate distance}

As a first step, PLD was performed at different distances $d$ to determine how close target and substrate have to be to one another in order for particle plume impingement to occur. Figure 3 illustrates the resulting surface textures after PLD at a single pulse peak fluence $F_{0}=19.7 \mathrm{~J} / \mathrm{cm}^{2}$, an overscan number $n=16$, and a scanning velocity $v=0.3 \mathrm{~mm} / \mathrm{s}$ which corresponds to a pulse-per-spot number $P P S=147$.

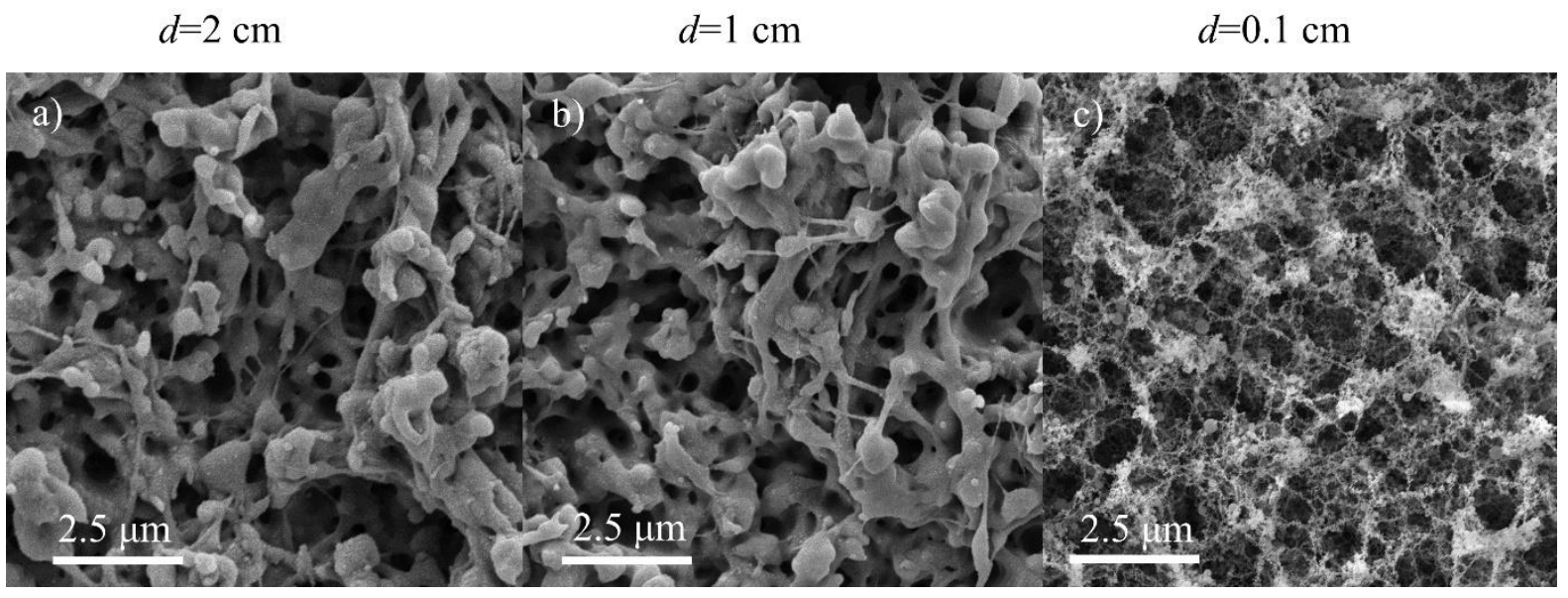

Figure 3: Effect of target-substrate distance on nanoparticle deposition with $\mathrm{F}_{0}=19.7 \mathrm{~J} / \mathrm{cm}^{2}, \mathrm{n}=16$, and v=0.3 $\mathrm{mm} / \mathrm{s}$. 
At $d=1$ and $2 \mathrm{~cm}$, which are distances that are in-line with classic low-pressure PLD operation, no nanoparticle coverage was observed. Nanoparticles only appeared at a target-substrate distance of $1 \mathrm{~mm}$. The requirement of a much smaller $d$ for atmospheric PLD can be explained by the plume dynamics during laser ablation. After a laser pulse is absorbed and ionisation of the target occurs, a plasma plume is formed that expands adiabatically until its pressure is equilibrated by that of the background gas [31]. The maximum plume length $L$ can then be described by the adiabatic expansion model:

$$
\begin{gathered}
L=A[(\gamma-1) E]^{\frac{1}{3 \gamma}} p^{\frac{-1}{3 \gamma}} V^{\frac{\gamma-1}{3 \gamma}} \\
A=\left(1+\frac{1}{\tan \theta}\right)\left(\frac{3 \tan \theta}{\pi+2 \pi \tan \theta}\right)^{\frac{1}{3}} \\
V=v \tau \omega
\end{gathered}
$$

Where $E$ is the laser pulse energy, $p$ is the background gas pressure, $V$ is the plasma volume at the end of the laser pulse, $v$ is the plasma expansion velocity, $\tau$ is the pulse duration, $\omega$ is the beam spot area, and $A$ is a geometrical factor that is dependent on the plume expansion angle $\theta$. Values of $v$ and $\theta$ were measured to be $4800 \mathrm{~m} / \mathrm{s}$ and $35^{\circ}$ respectively in a previous synchronised videography study of the expanding plume performed using our laser setup [32]. This results in a plasma length of $L=0.2 \mathrm{~mm}$. At this distance from the target, nanoparticles start to aggregate due to collisions with the background gas molecules and diffuse in the direction of the substrate. Amoruso et al. have shown that, in the case of fs ablation of metallic targets, the kinetic energy of the nanoparticles is still high enough that they are able to travel distances up to eight times farther than the plasma plume [33]. In our case, this corresponds to a distance of $1.6 \mathrm{~mm}$. Therefore, by using fs laser pulses and a setup that allows us to place the substrate within 
the range of the nanoparticle plume, PLD was successful without the need for lowpressure conditions.

Furthermore, the shape of the nanoparticles that were deposited was of interest since they formed a web-like structure consisting of nanochain wires linked through agglomerate nodes. Sivakumar et al. have demonstrated the formation of the same type of nanoparticle web by ablating titanium [34]. Their transmission electron microscopy (TEM) analysis showed that these nanochains are formed by individual nanoparticles fused together by an oxide layer formed by reaction with air. The authors were only able to create such structures by operating in the $\mathrm{MHz}$ regime which they dubbed as the frequency threshold for nanoweb formation. However, our study shows that this type of nanoparticle structure can be achieved at $1 \mathrm{kHz}$. Their proposed hypothesis for the frequency dependence of the microstructure formation is that, during femtosecond laser ablation, nanoparticles are mostly formed through vapour condensation on a picosecond timescale while agglomerates form by particle collision in the expanding plume on a timescale of around $1 \mu \mathrm{s}$. Therefore, when ablating in the MHz regime, a constant supply of nanoparticles can be generated on a timescale shorter than that of aggregate formation. This greatly increases the collision probability and allows the formation of agglomerates that are much more ordered than the random stacking typically observed in the $\mathrm{Hz}$ and $\mathrm{kHz}$ regime. In our case, due to the extremely small distance between target and substrate, there is a large degree of interaction between the yet-expanding plume and the flux of nanoparticles that have already reached the surface and are being sputtered back in the opposite direction. This greatly increases the collision probability inside the plume which explains why we observed nanochain formation at a much lower repetition rate.

\subsection{Optimisation of scanning velocity and overscan Number}


The next step was to optimise the scanning velocity and overscan number in order to minimise the time required for PLD while still achieving an acceptable amount of nanoparticle incorporation. Figure 4 illustrates the resulting microstructures $\left(F_{0}=19.7\right.$ $\left.\mathrm{J} / \mathrm{cm}^{2}\right)$.
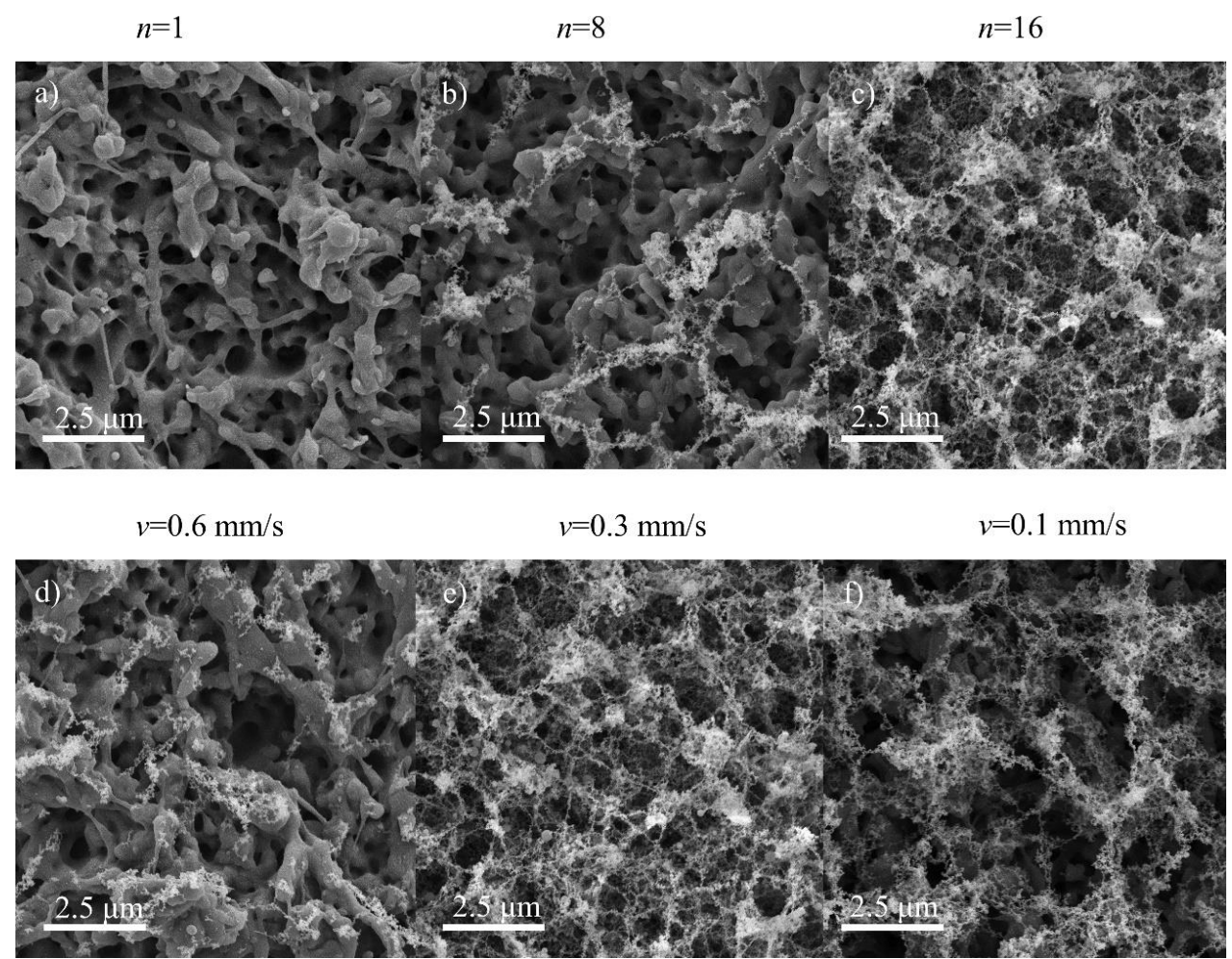

Figure 4: Effect of overscan number and scanning velocity on nanoparticle deposition.

No nanoparticle coverage was observed for $n=1$, while the coverage was very limited for $n=2$ despite the high operating fluence. This indicates that very high ablation depths and therefore large quantities of material ablation are required for successful deposition which again is due to the high collision frequency required for nanoweb formation. As for the effect of scanning velocity, limited coverage was observed at $v=0.6$ $\mathrm{mm} / \mathrm{s}$. Interestingly, operating at a very low scanning speed $(\nu=0.1 \mathrm{~mm} / \mathrm{s})$ decreased the nanoweb homogeneity with the network being characterised by larger, less interconnected agglomerates. This is somewhat counterintuitive as a lower scanning speed increases the PPS number and thus the ablation depth. One possibility is that the 
now very high flux of impinging nanoparticles starts sputtering the already deposited film thus causing the less agglomerated regions to break off. Following these results, $n=16$ and $v=0.3 \mathrm{~mm} / \mathrm{s}$ were chosen as operating parameters for the rest of the experiments.

\subsection{Effect of nanoparticle coverage on wettability}

After establishing the process parameters for successful fs PLD of titanium onto micromachined PET, we investigated the possibility of tuning wettability by controlling the density of deposited nanoparticles. Pristine PET has a mildly hydrophobic advancing contact angle $\left(\theta_{A}\right)$ of $92 \pm 2^{0}$ and a hydrophilic receding contact angle $\left(\theta_{R}\right)$ of $57 \pm 2^{0}$. After micromachining, the porous PET surface structure is more hydrophilic than the pristine PET with a $\theta_{A}$ of $82 \pm 2^{0}$ and a $\theta_{R}$ of $43 \pm 2^{0}$. This is in line with Wenzel theory since the increase in liquid-solid contact area induced by the large increase in roughness and porosity after machining enhances the intrinsic hydrophilicity of the material. There is also the possibility of a laser-induced increase in polar surface functional groups since photochemical reactions have been reported to occur on polymer surfaces during femtosecond laser micromachining [35]. However, our XPS analysis showed no significant difference in composition before and after machining as the atomic carbon-tooxygen ratio was close to the stoichiometric value of $2.5: 1$. Figure 5 presents the nanoparticle coverage and the resulting contact angles as a function of PLD operating fluence. 


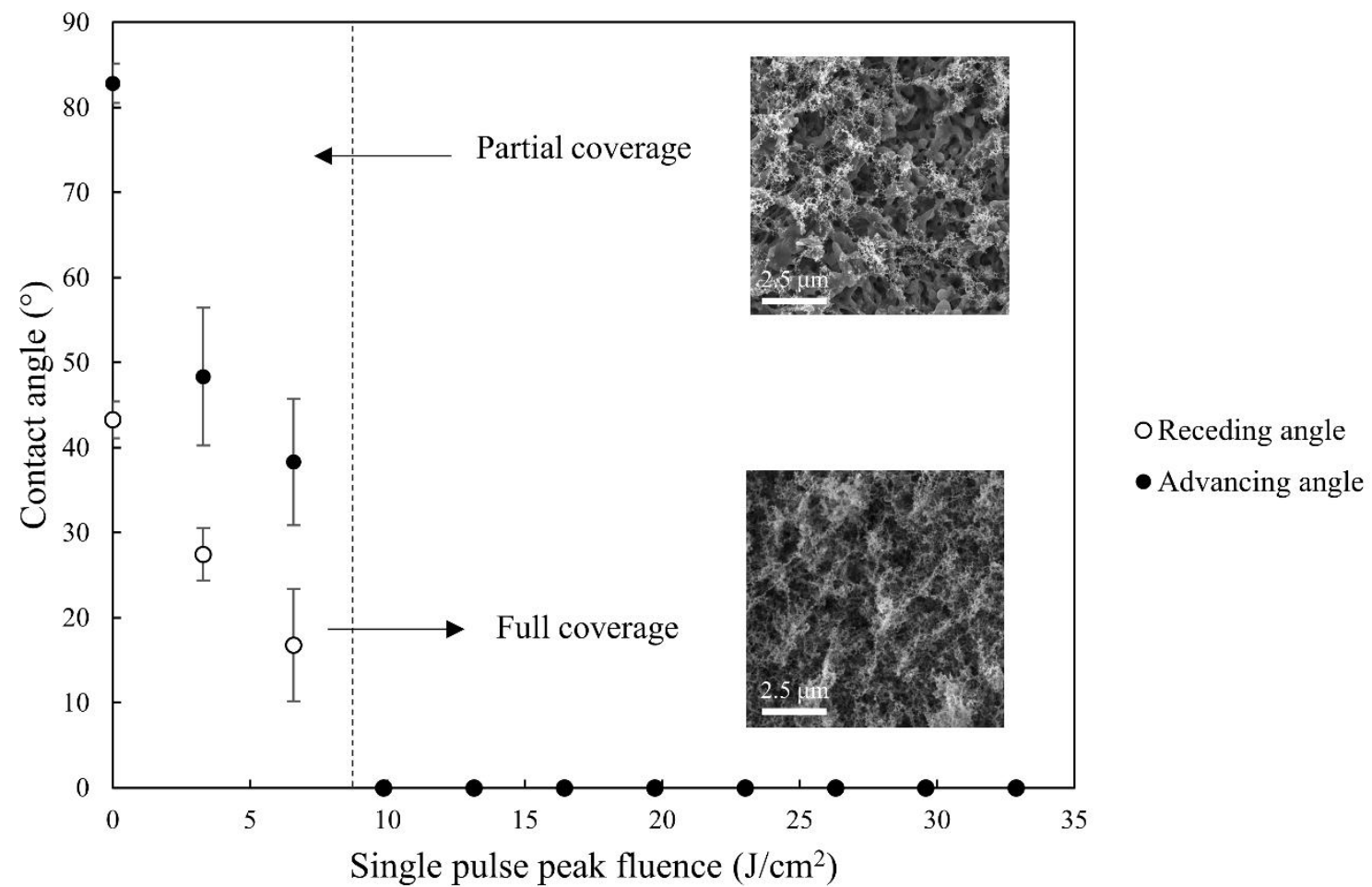

Figure 5: Surface coverage and contact angles as a function of fluence. Error bars represent the standard deviation of three measurements. Contact angle values of $0^{0}$ represent cases where wetting was so prominent that the full extent of the sample was covered by the water droplet. They do not represent actual measurements.

For fluences below $F_{0}=10 \mathrm{~J} / \mathrm{cm}^{2}$, nanochains are only deposited in between the substrate features which results in a partial coverage regime. ARCA measurements on these two samples demonstrated a significant increase in hydrophilicity in comparison with the micromachined sample. This decrease in measured contact angles correlates with increasing fluence. On the other hand, for operation at fluences above $F_{0}=10 \mathrm{~J} / \mathrm{cm}^{2}$, complete surface coverage by a heavily interconnected nanoweb was observed. This led to the water droplets completely wetting the sample surface and therefore rendering ARCA measurements impossible. This extremely hydrophilic behaviour is initially surprising as this very large degree of porosity and nanoroughness is usually expected to favour air trapping and therefore Cassie state wetting. Yet, the observed hydrophilicity can be explained by considering both the chemistry and topography of the deposited nanoweb. The SEMs show that the nanoweb structure exhibits features at different heights, as seen from the bright nodes which are located higher than the darker ones. Since the distance between these highest agglomerate nodes is varying and considerably 
larger than the average pore size, sagging of the water-air interface of a deposited droplet occurs. Thus, the sagged triple line gets in closer proximity to the lower located nodes which, due to the polar nature of the titanium oxide molecules, attract the sagging water interface to a degree that overcomes surface tension. The resulting increase in liquid-solid contact with the highly hydrophilic nanoparticles leads to complete wetting of the nanoweb. As a next step, all samples were subjected to vigorous sonication in an ethanol bath in order to assess the stability of nanoparticle incorporation into the substrates.

\subsection{Stability of nanoparticle incorporation}

Figure 6 presents the resulting microstructure after sonication. The SEM corresponding to $F_{0}=6.6 \mathrm{~J} / \mathrm{cm}^{2}$ clearly shows that the nanochains deposited in the partial coverage regime were not solidly attached to the surface as no nanoparticles remained after sonication. However, for fluences higher than $F_{0}=10 \mathrm{~J} / \mathrm{cm}^{2}$, an increasing amount of small spherical nanoparticle clusters was observed. This implies that the full coverage by the nanochain web observed before sonication constituted a weakly attached outer layer that was growing on top of the clusters that are actually strongly attached to the surface. Furthermore, the results shown in Figure 6 clearly demonstrate that nanoparticle coverage density can be controlled by operating fluence as the coverage observed ranged anywhere from light decoration of surface features $\left(F_{0}=13.2 \mathrm{~J} / \mathrm{cm}^{2}\right)$, to full surface coating that follows the texture of the substrate $\left(F_{0}=19.3 \mathrm{~J} / \mathrm{cm}^{2}\right)$, to the obstruction of the original pore structure by thick layer deposition $\left(F_{0}=32.9 \mathrm{~J} / \mathrm{cm}^{2}\right)$. The fact that surface coverage is correlated to fluence is due to the fact that, at higher energies, the kinetic energy of the nanoparticle plume is much higher meaning that a larger portion of impinging particles will adsorb to the substrate. 


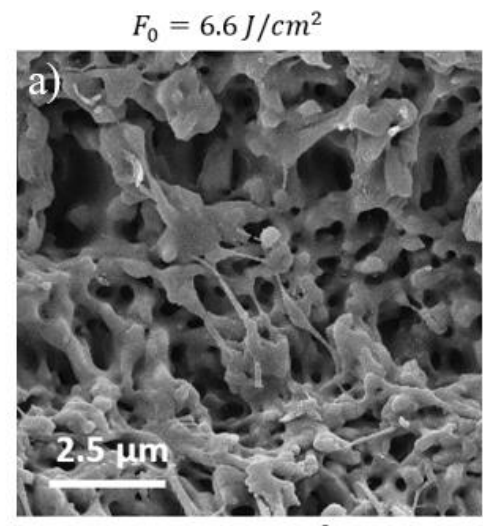

$F_{0}=26.3 \mathrm{~J} / \mathrm{cm}^{2}$

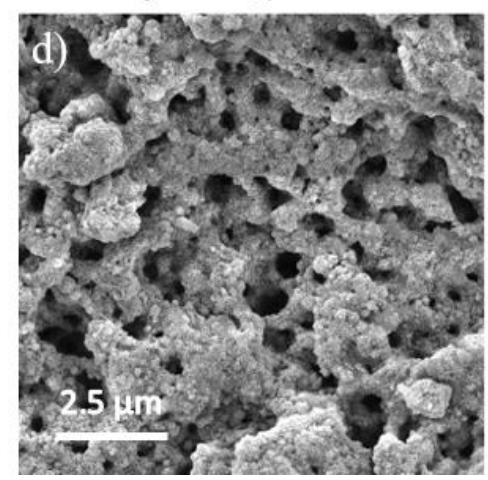

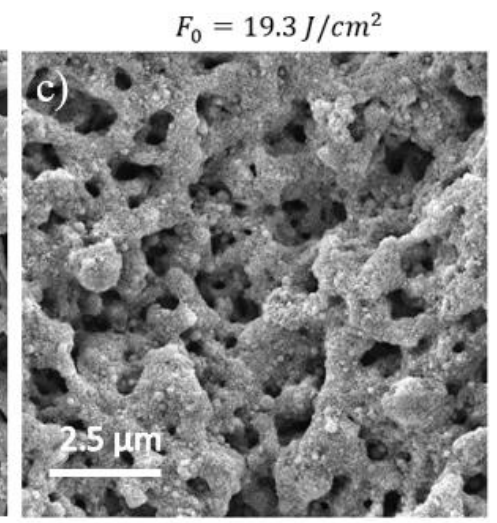

$F_{0}=32.9 \mathrm{~J} / \mathrm{cm}^{2}$

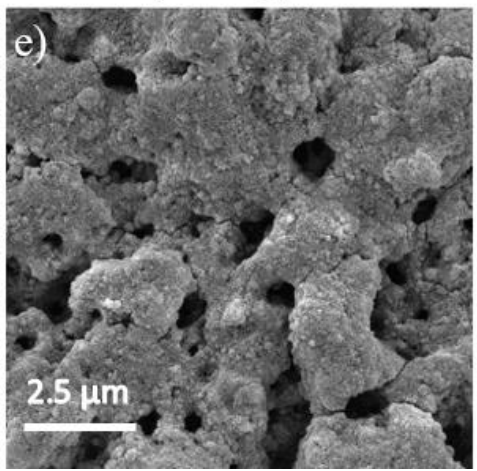

Figure 6: Evolution of remaining nanoparticle coverage as a function of fluence after sonication in ethanol.

As shown in Figure 7, the decrease in contact angle correlates very well with the increase in nanoparticles firmly attached to the surface. Below $F_{0}=10 \mathrm{~J} / \mathrm{cm}^{2}$, samples that observed nanochain incorporation and were significantly more hydrophilic than the original substrate before sonication now observed little change in wettability due to the removal of weakly adhered nanoparticles by sonication. Between $F_{0}=10 \mathrm{~J} / \mathrm{cm}^{2}$ and $F_{0}=19.3 \mathrm{~J} / \mathrm{cm}^{2}$, wettability variation is clearly achieved as the samples are increasingly hydrophilic with an almost linear decrease in advancing contact angle owing to the steady increase in nanoparticle coverage density. Finally, once titanium nanoparticles cover the entirety of the underlying microstructure, complete wetting is achieved again. In conclusion, these results demonstrate that any wetting behaviour between that of the original substrate surface and the fully wetted one can be easily achieved by finely controlling nanoparticle deposition through operating fluence adjustment. 


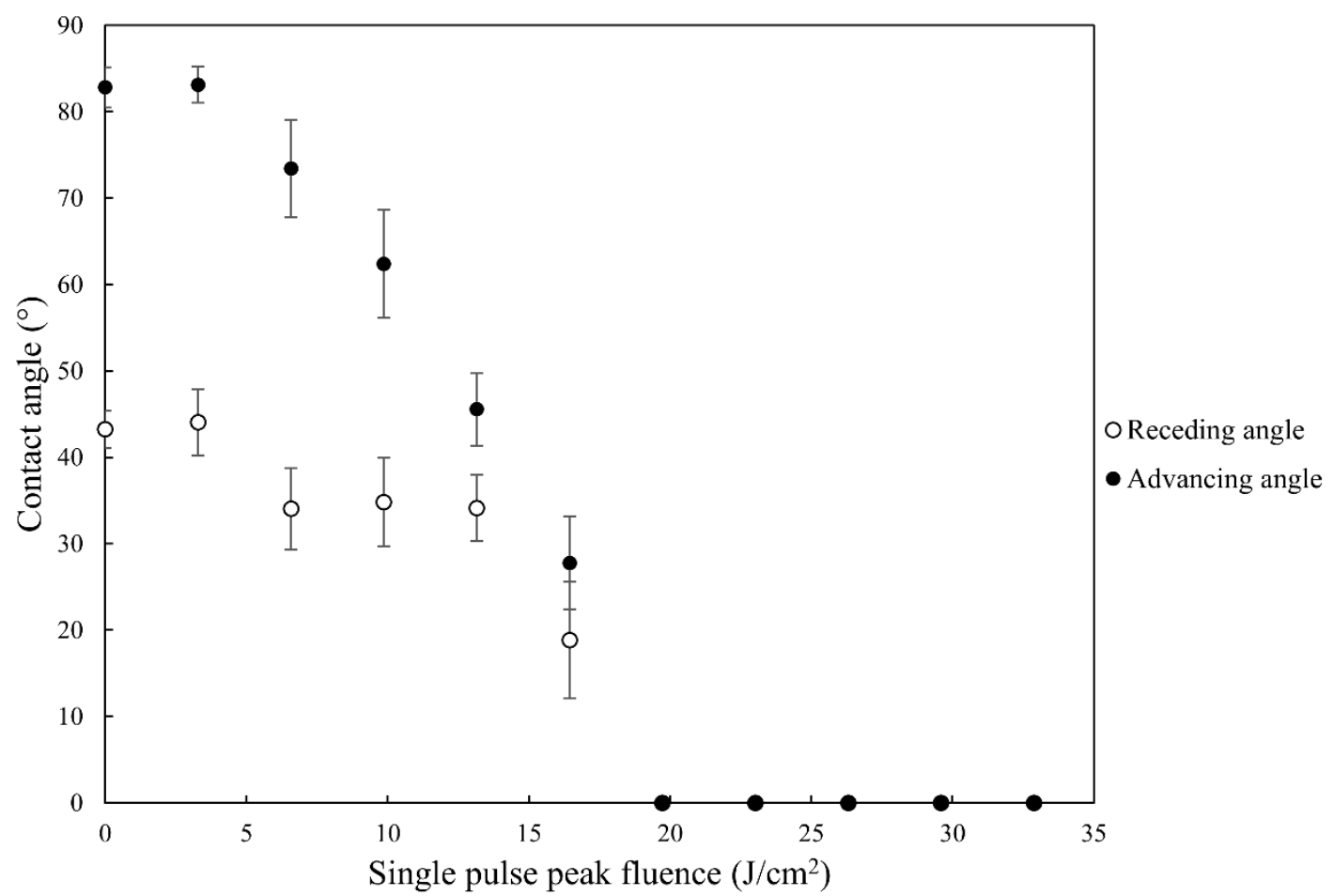

Figure 7: Evolution of contact angles as a function of fluence. Error bars represent the standard deviation of three measurements. Contact angle values of $0^{0}$ represent cases where wetting was so prominent that the full extent of the sample was covered by the water droplet. They do not represent actual measurements.

Finally, the evolution of surface chemistry with the increasing nanoparticle coverage was measured via XPS as presented in Figure 8. A clear increase in titanium content which correlates with the observed increase in nanoparticle coverage is observed. The accompanying increase in oxygen and sharp decrease in carbon suggests that titanium is indeed being deposited as an oxide. This was confirmed by the high-resolution spectra as illustrated in Figure 9. The spectrum of titanium clearly shows the presence of $\mathrm{TiO}_{2}$ as it is composed of its Ti2 $\mathrm{p}_{3 / 2}(458.5 \mathrm{eV})$ and $\mathrm{Tip}_{1 / 2}(464.5 \mathrm{eV})$ spin-orbital components as well as the typical satellite feature at $472 \mathrm{eV}$. This is further confirmed by the oxygen spectrum which is composed of a convoluted peak at $533 \mathrm{eV}$ which represents the single and double carbon-oxygen bonds and a large peak at $530 \mathrm{eV}$ which is typical of metallic oxides. From the peak areas, the ratio of oxygen-titanium to oxygen-carbon bonds was calculated to be 1.8. Knowing the composition of the sample and that of the original substrate, the fraction of oxygen that was not originally on the surface can be estimated 
to be 29 at\% which suggests a stoichiometric oxidation of the nanoparticles which is typical for PLD operation in an oxygen environment [36].

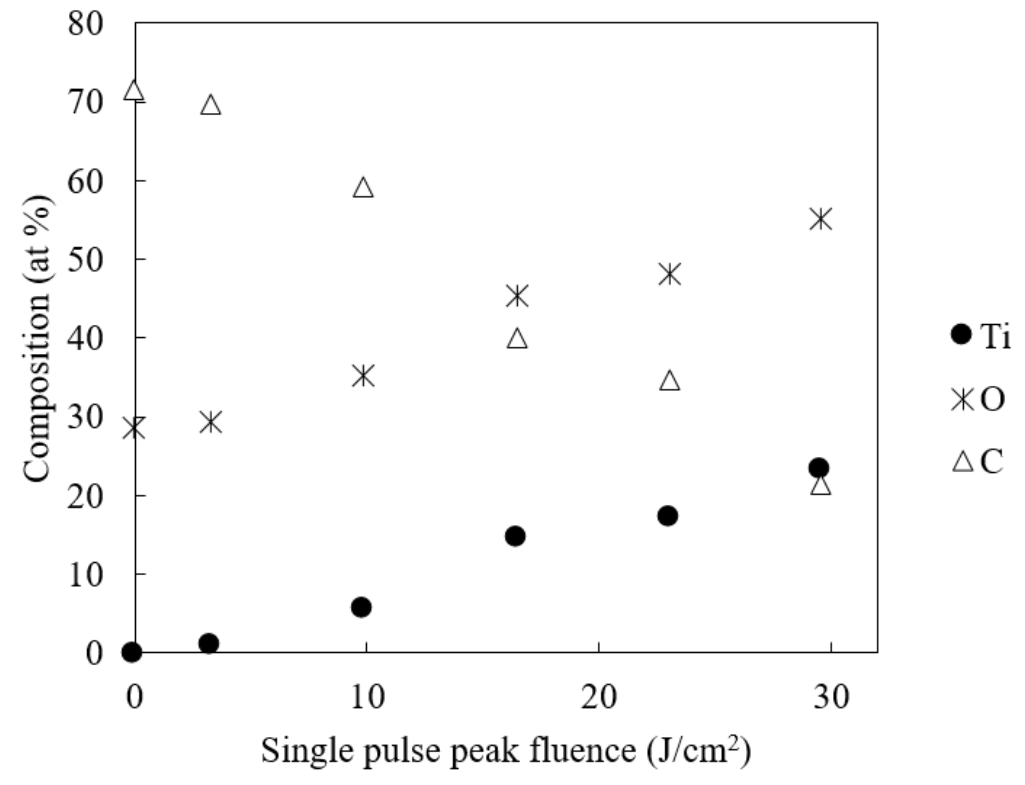

Figure 8: Evolution of surface chemistry as a function of fluence.
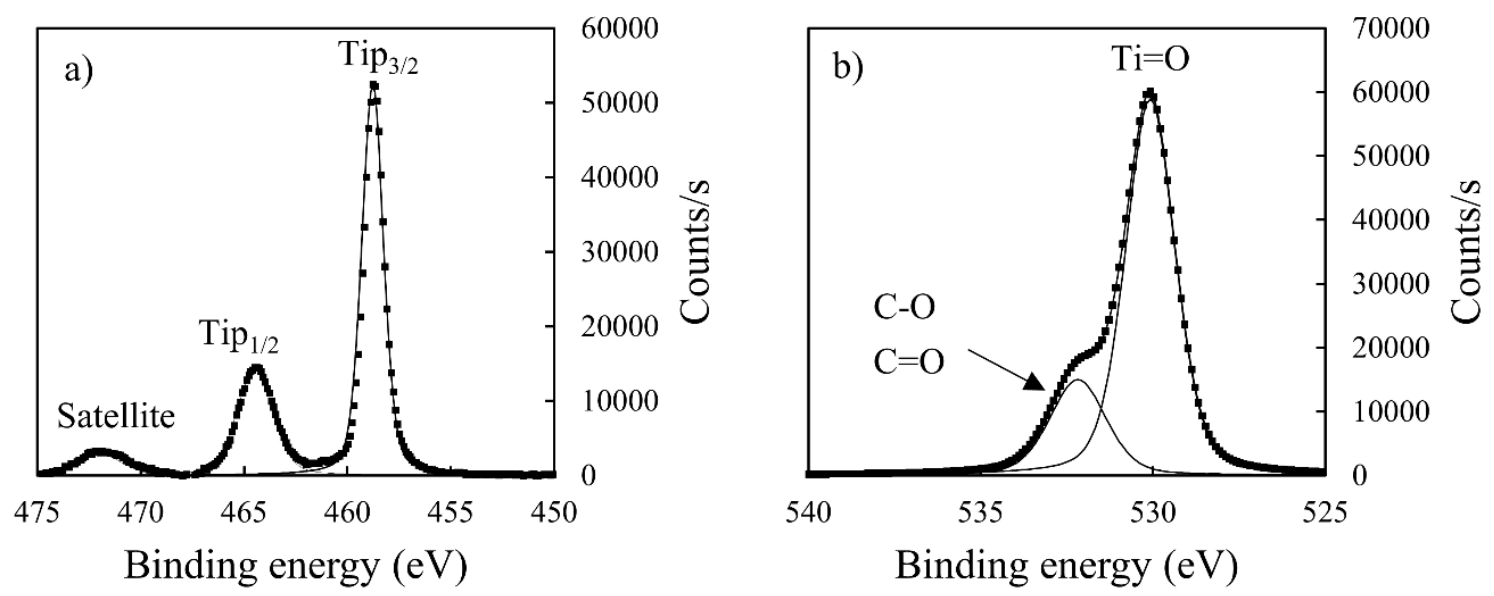

Figure 9: High-resolution spectra of a) $2 p$ sublevel of titanium and b) 1s sublevel of oxygen after PLD at $F_{0}=16.4$ $\mathrm{J} / \mathrm{cm}^{2}$.

Thus, by introducing controlled amounts of nanoparticles which are very hydrophilic in nature, we have created a composite surface with a controllable composition. Going back to Cassie-Baxter theory, this means that this novel technique achieves wettability variation by taking advantage of both the nanoroughness and the inherent wettability of the added nanoparticles without compromising the structure of the underlying porous substrate. 


\section{Conclusions}

Through the successful and controllable incorporation of titanium nanoparticles into a porous PET microstructure by pulsed laser deposition, successful variation of wettability was achieved while preserving the substrate topography. The use of a femtosecond laser source, coupled with the use of a $1 \mathrm{~mm}$ target-substrate distance, allowed the successful impingement of the nanoparticle plume on the substrate at atmospheric conditions. The traditional need of PLD to operate in vacuum was therefore circumvented. Increased collision probability in the plume due to recoil from the substrate led to the formation of an extremely hydrophilic titanium dioxide nanoweb. Sonication in an ethanol bath led to the detachment of the nanoweb revealing nanoparticle clusters that were strongly attached to the substrate. The inclusion of these clusters created a composite surface with controllable composition. Due to the inherent nanoroughness and chemical hydrophilicity of this added component, successful wettability variation was achieved. This work therefore highlights atmospheric pulsed laser deposition using a femtosecond laser as a simple and vacuum-free process for the modification of the wetting properties of hierarchical and high surface area polymers without compromising their topology. The practicality of this technique is further compounded by the fact that it requires the same equipment as the machining process with which the substrates are initially prepared.

\section{Funding information}

This work was supported by the National Sciences and Research Council of Canada (NSERC).

\section{Conflicts of Interest}

The authors declare no conflicts of interest. 


\section{References}

[1] P. Kaur, J.T. Hupp, S.T. Nguyen, Porous organic polymers in catalysis: opportunities and challenges, ACS Cat., 1 (2011) 819.

[2] B. Mattiasson, A. Kumar, I. Galaev, Macroporous polymers : production properties and biotechnological/biomedical applications, CRC Press/Taylor \& Francis, Boca Raton, 2010 .

[3] D.M. Cox, High surface area materials, in: Nanostructure Science and Technology: R\&D Status and Trends in Nanoparticles, Nanostructured Materials, and Nanodevices, Springer Netherlands, Dordrecht, 1999, pp. 49.

[4] F. Liang, J. Lehr, L. Danielczak, R. Leask, A.-M. Kietzig, Robust non-wetting PTFE surfaces by femtosecond laser machining, Int. J. Mol. Sci., 15 (2014) 13681.

[5] Y. Assaf, A.-M. Kietzig, Formation of porous networks on polymeric surfaces by femtosecond laser micromachining, in: Proc. SPIE 10092, 2017, pp. 100920X.

[6] T.A. Otitoju, A.L. Ahmad, B.S. Ooi, Superhydrophilic (superwetting) surfaces: A review on fabrication and application, J of Ind. and Eng. Chem., 47 (2017) 19.

[7] R.N. Wenzel, Resistance of solid surfaces to wetting by water, Ind. Eng. Chem., 28 (1936) 988.

[8] K. Akira, N. Hitoshi, Wetting behavior of liquid on geometrical rough surface formed by photolithography, JaJAP, 33 (1994) L1283.

[9] L. Barbieri, E. Wagner, P. Hoffmann, Water Wetting Transition Parameters of Perfluorinated Substrates with Periodically Distributed Flat-Top Microscale Obstacles, Langmuir, 23 (2007) 1723-1734.

[10] Y. Kameya, Wettability modification of polydimethylsiloxane surface by fabricating micropillar and microhole arrays, MatL, 196 (2017) 320-323.

[11] A. Milionis, D. Fragouli, I.S. Bayer, A. Athanassiou, Water Adhesion to LaserTreated Surfaces, in: Laser Surface Modification and Adhesion, John Wiley \& Sons, Inc., 2014, pp. 377-413.

[12] D.G. Waugh, J. Lawrence, On the use of $\mathrm{CO} 2$ laser induced surface patterns to modify the wettability of poly(methyl methacrylate) (PMMA), Opt. L. E., 48 (2010) 707715.

[13] J. Yong, F. Chen, Q. Yang, D. Zhang, H. Bian, G. Du, J. Si, X. Meng, X. Hou, Controllable Adhesive Superhydrophobic Surfaces Based on PDMS Microwell Arrays, Langmuir, 29 (2013) 3274-3279.

[14] F. Arefi-Khonsari, M. Tatoulian, F. Bretagnol, O. Bouloussa, F. Rondelez, Processing of polymers by plasma technologies, SuCT, 200 (2005) 14-20. 
[15] D. Pappas, A. Bujanda, J.D. Demaree, J.K. Hirvonen, W. Kosik, R. Jensen, S. McKnight, Surface modification of polyamide fibers and films using atmospheric plasmas, SuCT, 201 (2006) 4384-4388.

[16] J.A. Juárez-Moreno, A. Ávila-Ortega, A.I. Oliva, F. Avilés, J.V. Cauich-Rodríguez, Effect of wettability and surface roughness on the adhesion properties of collagen on PDMS films treated by capacitively coupled oxygen plasma, Appl. Surf. Sci., 349 (2015) 763-773.

[17] A.B.D. Cassie, S. Baxter, Wettability of porous surfaces, Trans. Faraday Society, 40 (1944) 546.

[18] N. Gao, Y. Yan, Characterisation of surface wettability based on nanoparticles, Nanoscale, 4 (2012) 2202.

[19] G. Malandrino, Chemical vapour deposition. precursors, processes and applications., Angew. Chem. Int. Ed., 48 (2009) 7478.

[20] M. Sanz, M. Walczak, R. de Nalda, M. Oujja, J.F. Marco, J. Rodriguez, J.G. Izquierdo, L. Bañares, M. Castillejo, Femtosecond pulsed laser deposition of nanostructured TiO2 films, Appl. Surf. Sci., 255 (2009) 5206.

[21] M.N.R. Ashfold, F. Claeyssens, G.M. Fuge, S.J. Henley, Pulsed laser ablation and deposition of thin films, ChSRv, 33 (2004) 23.

[22] F. Garrelie, C. Donnet, A.-S. Loir, N. Benchikh, New trends in femtosecond pulsed laser deposition and femtosecond produced plasma diagnostics, Proc. SPIE, (2006).

[23] Z. Yang, J. Hao, Progress in pulsed laser deposited two-dimensional layered materials for device applications, J of Mat. Chem. C, 4 (2016) 8859-8878.

[24] D. Fischer, A. von Mankowski, A. Ranft, S.K. Vasa, R. Linser, J. Mannhart, B.V. Lotsch, ZIF-8 Films Prepared by Femtosecond Pulsed-Laser Deposition, Chem. Mater., 29 (2017) 5148-5155.

[25] T. Kononenko, V. Konov, E. Lubnin, F. Dausinger, Pulsed laser deposition of hard carbon coatings at atmospheric pressure, Quantum Electronics, 33 (2003) 189.

[26] N. Nedyalkov, A. Nikolov, P. Atanasov, M. Alexandrov, M. Terakawa, H. Shimizu, Nanostructured $\mathrm{Au}$ film produced by pulsed laser deposition in air at atmospheric pressure, Opt. \& La.Tech., 64 (2014) 41.

[27] M. Ronán, H. Cian, B. Komal, S. Apryll, V. Mercedes, B. Dermot, Pulsed laser deposition of plasmonic nanostructured gold on flexible transparent polymers at atmospheric pressure, J. Phys. D: Appl. Phys., 50 (2017) 245303.

[28] M.J. Aziz, Film growth mechanisms in pulsed laser deposition, Appl. Phys. A, 93 (2008) 579. 
[29] B.L. Zhu, X.H. Sun, X.Z. Zhao, F.H. Su, G.H. Li, X.G. Wu, J. Wu, R. Wu, J. Liu, The effects of substrate temperature on the structure and properties of $\mathrm{ZnO}$ films prepared by pulsed laser deposition, Vacuu, 82 (2008) 495.

[30] T. Donnelly, B. Doggett, J.G. Lunney, Pulsed laser deposition of nanostructured Ag films, Appl. Surf. Sci., 252 (2006) 4445-4448.

[31] S.S. Harilal, C.V. Bindhu, M.S. Tillack, F. Najmabadi, A.C. Gaeris, Internal structure and expansion dynamics of laser ablation plumes into ambient gases, JAP, 93 (2003) 2380-2388.

[32] S. Paolasini, A. Kietzig, Synchronized videography of plasma plume expansion during femtosecond laser ablation, in: Proc. SPIE 8967, 2014, pp. 896710.

[33] S. Amoruso, R. Bruzzese, X. Wang, J. Xia, Propagation of a femtosecond pulsed laser ablation plume into a background atmosphere, ApPhL, 92 (2008) 041503.

[34] M. Sivakumar, V. Krishnan, B. Tan, Study of metallic fibrous nanoparticle aggregate produced using femtosecond laser radiation under ambient conditions, Nanot, 21 (2010) 225601.

[35] Z.K. Wang, H.Y. Zheng, C.P. Lim, Y.C. Lam, Polymer hydrophilicity and hydrophobicity induced by femtosecond laser direct irradiation, ApPhL, 95 (2009) 111110.

[36] J.A. De Mesa, A.M. Amo, J.J.C. Miranda, H.O. Salazar, R.V. Sarmago, W.O. Garcia, Effects of deposition pressure and target-substrate distance on growth of $\mathrm{ZnO}$ by femtosecond pulsed laser deposition, J of Las. Micro Nanoeng., 11 (2016) 21. 